Check for updates

Cite this: Chem. Sci., 2018, 9, 8099

¿ All publication charges for this article have been paid for by the Royal Society of Chemistry

Received 17th July 2018

Accepted 25th August 2018

DOI: $10.1039 / \mathrm{c} 8 \mathrm{sc} 03168 \mathrm{a}$

rsc.li/chemical-science

\section{Near infrared excitation and emission in rare earth MOFs via encapsulation of organic dyes $\uparrow$}

\author{
Chong Liu, (D) a Svetlana V. Eliseeva, (D) ${ }^{b}$ Tian-Yi Luo, (D) a Patrick F. Muldoon, ${ }^{a}$ \\ Stéphane Petoud (iD *ab and Nathaniel L. Rosi (D) *a
}

\begin{abstract}
We successfully demonstrate that metal-organic frameworks (MOFs) can be designed to be excited and emit within the biological diagnostic window $(650-1450 \mathrm{~nm})$. An isoreticular series of anionic rare earth MOFs with fcu topology was synthesized using 10 different rare earth elements $\left(\mathrm{Y}^{3+}, \mathrm{Eu}^{3+}, \mathrm{Gd}^{3+}, \mathrm{Tb}^{3+}\right.$, $\left.\mathrm{Dy}^{3+}, \mathrm{Ho}^{3+}, \mathrm{Er}^{3+}, \mathrm{Tm}^{3+}, \mathrm{Yb}^{3+}, \mathrm{Lu}^{3+}\right)$ and common linear ditopic ligands. Five different cationic dye molecules were incorporated into the MOFs via ion exchange. When LDS 750, which exhibits lowenergy absorption in the range $450-770 \mathrm{~nm}$, is loaded into an $\mathrm{Yb}^{3+}$-MOF, it can be used as an antenna to sensitize the near-infrared (NIR) emission of $\mathrm{Yb}^{3+}$ centred at $980 \mathrm{~nm}$.
\end{abstract}

\section{Introduction}

Biological tissues exhibit low autofluorescence and low absorption in the so-called biological diagnostic window (650$1450 \mathrm{~nm}$ ). Biological imaging agents that both absorb and emit in this biological window can allow achievement of (i) higher sensitivity because their signals can be easily discriminated from the background and (ii) deeper tissue penetration due to minimal or no overlap of their excitation window with tissue absorption. ${ }^{1-3}$

Trivalent rare earth $\left(\mathrm{RE}^{3+}\right)$ based compounds emitting in the near-infrared (NIR) offer complementary advantages for biological imaging applications. ${ }^{4} \mathrm{RE}^{3+}$ emitters exhibit narrow emission bands, long luminescence lifetimes ( $\mu$ s-ms range), and high resistance to photobleaching. In addition, their emission signals are not affected by environmental factors and experimental conditions. However, the design of $\mathrm{RE}^{3+}$ compounds with intense luminescence can be challenging because: (i) the direct excitation of $\mathrm{RE}^{3+}$ is inefficient due to the low absorbance of free $\mathrm{RE}^{3+}$ as most of the $\mathrm{f} \rightarrow \mathrm{f}$ transitions are forbidden by the Laporte rule; and (ii) overtones of $\mathrm{C}-\mathrm{H}, \mathrm{O}-\mathrm{H}$, and $\mathrm{N}-\mathrm{H}$ vibrations can cause non-radiative deactivation and decreased intensities of $\mathrm{RE}^{3+}$ signals. To overcome these challenges, $\mathrm{RE}^{3+}$ electronic states must be indirectly populated by a photonic converter, such as an organic chromophore with high extinction coefficient, in a sensitizing process named the

${ }^{a}$ Department of Chemistry, University of Pittsburgh, Pittsburgh, PA 15260, USA. E-mail: nrosi@pitt.edu

${ }^{b}$ Centre de Biophysique Moléculaire, CNRS UPR 4301, 45071 Orléans, France. E-mail: stephane.petoud@inserm.fr

$\uparrow$ Electronic supplementary information (ESI) available: Synthetic details, general characterization, X-ray crystallography, photoluminescence studies, additional figures and tables. CCDC 1840493-1840495. For ESI and crystallographic data in CIF or other electronic format see DOI: $10.1039 / \mathrm{c} 8 \mathrm{sc} 03168 \mathrm{a}$ 'antenna effect'.5,6 Antenna molecules possessing suitable electronic structures must be placed in sufficiently close proximity to $\mathrm{RE}^{3+}$ to provide efficient sensitization through energy transfer, as well as to ensure good protection against the vibrational overtones from external molecules. For biological imaging applications, especially in vivo, a combination of both NIR-emitting $\mathrm{RE}^{3+}$ and antennae that absorb in the lower energy range (visible or NIR) would be necessary.

We have been developing $\mathrm{RE}^{3+}$-based metal-organic framework (MOF) materials to study and harness their luminescent properties in the field of biological applications, motivated by the ultimate goal of in vivo imaging. ${ }^{7-11}$ MOFs are intriguing materials for harnessing $\mathrm{RE}^{3+}$ luminescence because they can provide: (i) a high density arrangement of $\mathrm{RE}^{3+}$ emitters and sensitizing species in proximity to one another; (ii) macroscopic entities and stable coordination spheres with spatially constrained $\mathrm{RE}^{3+}$ and ligands that effectively decrease undesirable interactions with extraneous species; and (iii) a highly diverse collection of structures with functional handles that can be exploited for a broad range of applications. ${ }^{12-14}$

Specifically, our research in this area has focused on the exploration of different strategies for optimizing the sensitization of $\mathrm{RE}^{3+}$-based luminescent MOFs, with the aim of achieving excitation in the biological diagnostic window. We have prepared a NIR-emitting $\mathrm{Yb}^{3+}$-based in vitro imaging agent using nano-sized MOFs comprising phenylene-based antennae and $\mathrm{Yb}^{3+} \cdot{ }^{10}$ More recently, we established a new strategy for expanding the $\pi$ conjugation of the MOF linkers via covalent postsynthetic modification in order to red-shift the antennae absorption and thus the $\mathrm{RE}^{3+}$ excitation wavelengths. ${ }^{11}$ Herein, we report a new strategy to red-shift the excitation wavelength of $\mathrm{RE}^{3+}$ MOFs via incorporation of organic molecular chromophores into MOF channels. ${ }^{15-17}$ 


\section{Results and discussion}

An isoreticular family of anionic fcu MOFs based on $\mathrm{RE}^{3+}{ }_{6}$ clusters was selected for the present study. ${ }^{18}$ Pioneered by Eddaoudi and co-workers, these MOFs are typically constructed using monofluorinated dicarboxylates, which direct the formation of the $\mathrm{RE}_{6}{ }^{3+}$ clusters. ${ }^{19-22}$ Here, we used 2,6-difluorobenzoic acid (DFBA) as a modulator to effect formation of the clusters and analogous fcu MOFs using non-fluorinated ligands including 2,6-naphthalenedicarboxylic acid ( $\mathrm{H}_{2}$-NDC), 1,1'biphenyl-4,4'-dicarboxylic acid ( $\mathrm{H}_{2}$-BPDC), and 4,4'-azobenzenedicarboxylic acid ( $\mathrm{H}_{2}$-ABDC) (Fig. 1), namely $\mathbf{D y}^{\mathbf{3 +}}$-NDC, $\mathbf{D y}^{\mathbf{3}^{+}}$BPDC, and $\mathbf{D y}^{3+}$-ABDC. These modulated MOF syntheses yielded large single crystals suitable for single crystal X-ray diffraction (SC-XRD) (Fig. S1†), and structures of the $\mathrm{Dy}^{3+}$ MOFs were successfully determined (Fig. 1c-e). Phase purity of the as-synthesized $\mathrm{Dy}^{3+}$-MOFs was assessed and confirmed by comparing simulated diffraction patterns to those collected via powder X-ray diffraction (PXRD) (Fig. S5 $\dagger$ ). The chemical formulae of the as-synthesized MOFs were determined by a combination of elemental microanalysis and thermogravimetric analysis (see ESI Section 2.8 for details). It is noteworthy

(a)

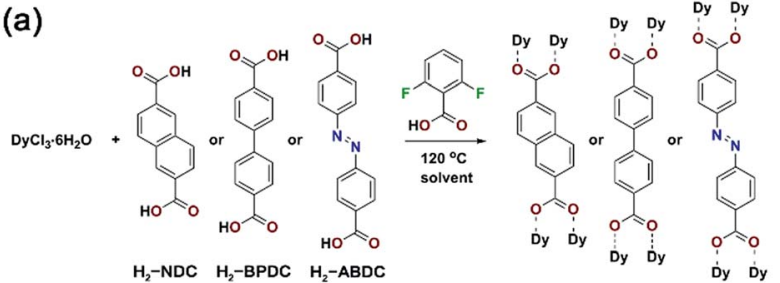

(b)

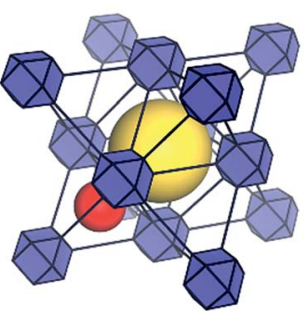

(d)

(c)

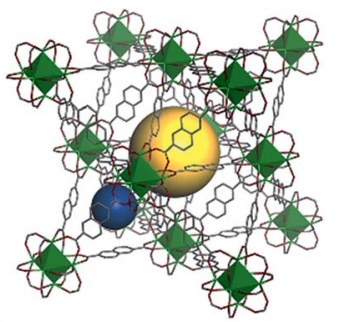

(e)
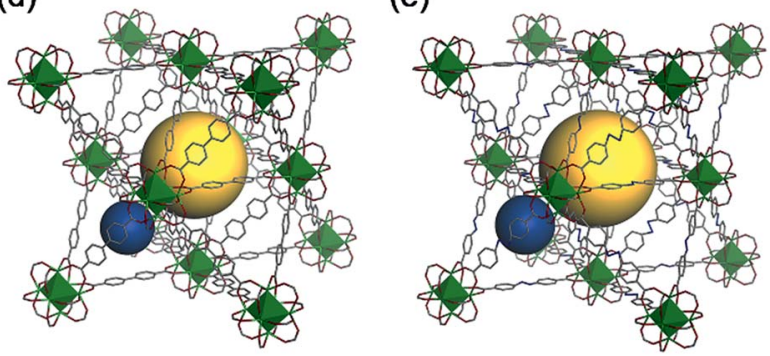

Fig. 1 (a) Reaction scheme of DFBA-modulated syntheses of $\mathrm{Dy}^{3+}$ NDC, $\mathrm{Dy}^{3+}-\mathrm{BPDC}$, and $\mathrm{Dy}^{3+}-\mathrm{ABDC}$. (b) Schematic representation of fcu topology with augmented nodes shown as purple cuboctahedra, an octahedral cavity and a tetrahedral cavity highlighted with yellow and red spheres, respectively. (c), (d) and (e) SC-XRD structures of $\mathrm{Dy}^{3+}-\mathrm{NDC}, \mathrm{Dy}^{3+}-\mathrm{BPDC}$, and $\mathrm{Dy}^{3+}-\mathrm{ABDC}$ : Dy $6\left(\mu_{3}-\mathrm{OH}\right)_{8}$, green octahedra; O, red sticks; $\mathrm{N}$, blue sticks; C, grey sticks; $\mathrm{H}$, omitted for clarity; octahedral cavities and tetrahedral cavities highlighted with yellow and blue spheres, respectively. that the as-synthesized MOFs do not contain DFBA modulator, as determined via ${ }^{1} \mathrm{H}$ nuclear magnetic resonance (NMR) spectroscopy of dissolved MOF samples (Fig. S9†). Although activation procedures were not optimized, $\mathrm{N}_{2}$ adsorption experiments revealed that $\mathbf{D y}^{3+}$-NDC, $\mathbf{D y}^{3+}$-BPDC, and $\mathbf{D y}^{3+}$ ABDC were each permanently porous (Fig. S10 $\dagger$ ).

DFBA-modulated conditions were found to be compatible with 9 other $\mathrm{RE}^{3+}\left(\mathrm{Y}^{3+}, \mathrm{Eu}^{3+}, \mathrm{Gd}^{3+}, \mathrm{Tb}^{3+}, \mathrm{Ho}^{3+}, \mathrm{Er}^{3+}, \mathrm{Tm}^{3+}, \mathrm{Yb}^{3+}\right.$, $\mathrm{Lu}^{3+}$ ) in addition to $\mathrm{Dy}^{3+}$. Isostructural MOFs were formed in pure phases, as determined by comparing their respective PXRD patterns with those of $\mathrm{Dy}^{3+}$-MOFs and simulated patterns from SC-XRD structures (Fig. S11-S13†). Such results confirm that the synthesized MOFs are a versatile platform for the development of luminescent materials with different functional properties.

The fcu $\mathrm{RE}^{3+}$-MOFs are anionic with dimethylammonium cations residing in the pores balancing the framework charges (see ESI Section 2.8 for details $\dagger$ ). Therefore, it is expected that the native cations can be exchanged with other positively charged species. In addition to the MOF ligands, a broader sensitization of the $\mathrm{RE}^{3+}$ centres could be obtained if cationic dyes absorbing at longer wavelengths were incorporated into the MOF channels. Ion exchange experiments were carried out with $\mathbf{D y}^{3+}$-NDC, $\mathbf{D y}^{3+}$-BPDC, and $\mathbf{D y}^{3+}$-ABDC, each having different pore dimensions, and five cationic dyes (Rhodamine 590, Rhodamine 610, Rhodamine 640, LDS 698, LDS 750). Space filling models of $\mathbf{D y}^{3+}$-BPDC and the five dyes are shown in Fig. 2a and b. After similar treatment with various dye solutions (Fig. S14 $\dagger$ ), the MOF crystallinity was retained in all cases, as indicated by PXRD patterns (Fig. S15-S17 $\dagger$ ). Different degrees of

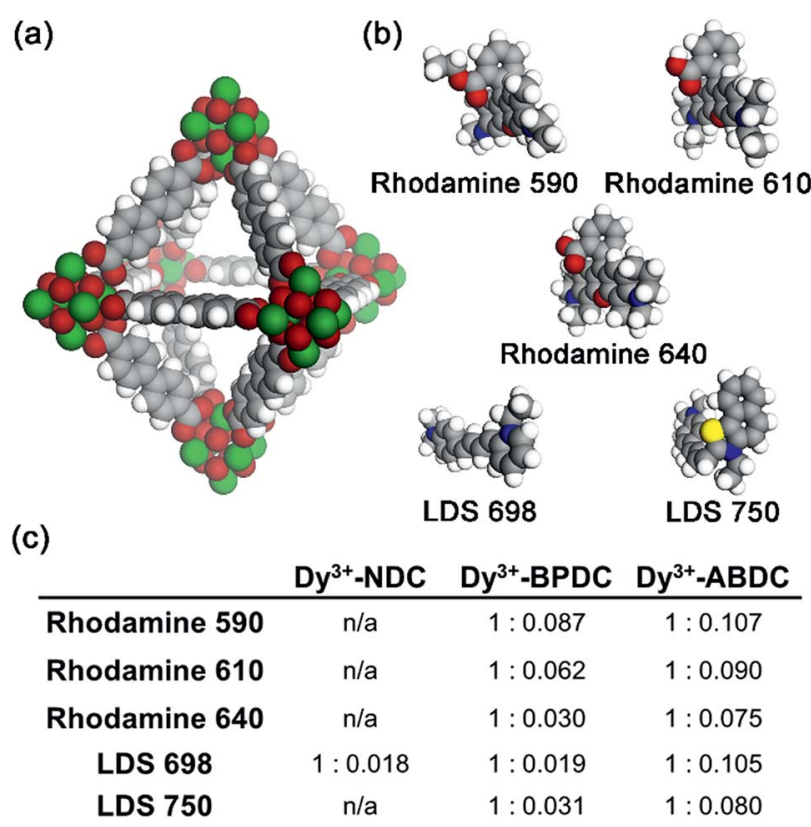

Fig. 2 (a) Space-filling model of the octahedral cavity of $\mathrm{Dy}^{3+}$-BPDC. (b) Space-filling models of the five dyes tested for cation exchange: Rhodamine 590, Rhodamine 610, Rhodamine 640, LDS 698, and LDS 750. (c) $\mathrm{Dy}^{3+}$ : dye ratios in different dyeaDy ${ }^{3+}$-MOFs based on ${ }^{1} \mathrm{H}$ NMR analysis of the composition. 
loading (Fig. 2c) were observed in $\mathbf{D y}^{3+}$-NDC, $\mathbf{D y}^{3+}$-BPDC, and $\mathbf{D y}^{3+}$-ABDC, as determined from ${ }^{1} \mathrm{H}$ NMR analysis of the dissolved products (Fig. S18-S31†).

Dye-loaded $\mathbf{R E}^{3+}$-BPDC was chosen for luminescence studies because it could adsorb a quantifiable amount of dye and because the dye molecules remain within the MOF channels after several cycles of washing. In contrast, only trace amounts of dyes could be incorporated into $\mathbf{R E}^{3+}$-NDC and significant quantities of dyes leached out of $\mathbf{R E}^{3+}$-ABDC upon washing, which could be due to unfavourable interactions between the dyes and the $\mathbf{R E}^{3+}$-ABDC MOFs. As previously articulated, having both antennae absorption and $\mathrm{RE}^{3+}$ emission in the biological diagnostic window is ideal for optical imaging experiments and diagnostics on biological materials, especially when carried out in vivo. On the basis of this criterion, LDS 750 was chosen as the sensitizer and was incorporated into $\mathbf{Y b}^{3+}$ BPDC. The ratio of $\mathrm{Yb}^{3+}$ to LDS 750 can be finely controlled by varying the loading condition (e.g. concentration, reaction time), as confirmed by ${ }^{1} \mathrm{H}$ NMR quantification (Fig. S32 $\dagger$ ) and summarized in Table S4. $\dagger^{\dagger}$

LDS 750@Yb $\mathbf{Y b}^{3+}$-BPDC $\left(\mathrm{Yb}^{3+}:\right.$ LDS $\left.750=1: 0.003\right)$ was then studied by optical spectroscopy. The emission spectrum recorded upon an excitation wavelength corresponding to low energy (600 nm, Fig. 3a, red curve) reveals the presence of a sharp emission band with an apparent maximum at $980 \mathrm{~nm}$ that can be attributed to $\mathrm{Yb}^{3+}$ located in the MOF structure. As the $\mathrm{Yb}^{3+}$ cation does not possess any accepting level below $980 \mathrm{~nm}$, the sensitization can only take place through the organic chromophores. LDS 750@ $\mathbf{Y b}^{3+}$-BPDC contains two different chromophores that could potentially act as sensitizers of the $\mathrm{Yb}^{3+}$ emission, through the 'antenna effect': (i) the BPDC linkers that constitute the MOF scaffold; and (ii) the LDS 750 encapsulated inside the MOF pores. The comparison of the excitation spectra recorded upon monitoring emission at $980 \mathrm{~nm}$ of $\mathbf{Y b}^{3+}$-BPDC and LDS 750@Yb ${ }^{3+}$-BPDC (Fig. 3b) revealed no bands above $600 \mathrm{~nm}$ for the former, while a broad and intense excitation band extended up to $800 \mathrm{~nm}$ was observed for the latter. Therefore, upon $600 \mathrm{~nm}$ excitation of LDS $750 @ \mathbf{Y b}^{3+}$-BPDC, only LDS 750 can act as the sensitizer of the characteristic $\mathrm{Yb}^{3+}$ emission. These results confirm the validity of the approach and the creation of a new MOF system capable of sensitizing NIR-emitting $\mathrm{RE}^{3+}$ with an extended range of excitation wavelengths through the selection of encapsulated chromophores.

It is also worth noting that despite the low amount of LDS 750 located in the MOF pores, it was sufficient to generate an $\mathrm{Yb}^{3+}$ signal that is intense enough to be not only monitored but also quantified. $\mathrm{Yb}^{3+}$-centred quantum yields recorded upon two excitation wavelengths corresponding to low and high energies as well as luminescence lifetimes were collected on both $\mathbf{Y b}^{3+}$-BPDC and LDS 750@Y $\mathbf{Y b}^{3+}$-BPDC (Fig. 3c) to quantify the effect of LDS 750 inside the MOF. Luminescence lifetime values allow us to obtain information about the environment around $\mathrm{Yb}^{3+}$. A more quenching environment implies a shorter luminescence lifetime. The results shown in Fig. 3c can be rationalized by the presence of two different environments around the $\mathrm{Yb}^{3+}$ : the longer luminescence lifetime value corresponds to the $\mathrm{Yb}^{3+}$ located at the core of the MOF crystals that may benefit from a higher level of protection from the environment (i.e. solvent molecules). The second, smaller lifetime component can be attributed to the $\mathrm{Yb}^{3+}$ that are present at or near the exterior of the MOF crystals which are more exposed to sources of non-radiative deactivation. It should be noted that the incorporation of LDS 750 inside the pores of $\mathbf{Y b}^{3+}$-BPDC has no significant impact on the $\mathrm{Yb}^{3+}$ luminescence lifetimes (i.e. the longer lifetime component remains the same, within experimental error), while the shorter lifetime component decreases by 1.4 times. Such results point out the similarities of the coordination environments around the $\mathrm{Yb}^{3+}$ in both MOFs.

$\mathrm{Yb}^{3+}$-centred quantum yield $(Q)$ values determined under excitation at 320 and $600 \mathrm{~nm}$ (Fig. 3a) reflect quantitatively the combination of the protection of $\mathrm{Yb}^{3+}$ against non-radiative deactivations and the sensitization efficiency of the chromophores (Fig. 3c). We have previously concluded from the luminescence lifetime measurements that the environments around $\mathrm{Yb}^{3+}$ are not strongly affected by the presence of LDS 750 . Quantum yields will therefore be mainly dependent on the sensitization efficiencies of the BPDC linker and LDS 750. Upon excitation at $320 \mathrm{~nm}$ (BPDC chromophore), the quantum yield (a)

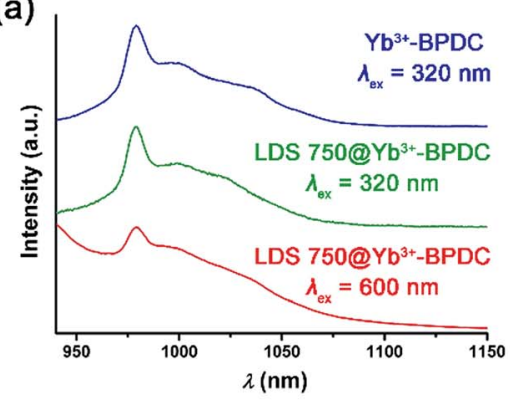

(b)

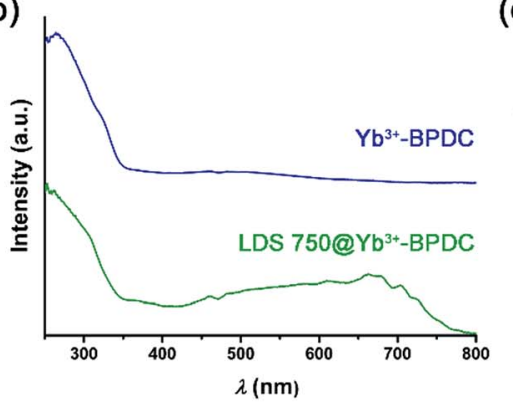

(c)

\begin{tabular}{cccc} 
& $\mathbf{Y b}^{3+}$-BPDC & \multicolumn{2}{c}{ LDS 750@Y $\mathbf{b}^{3+}$-BPDC } \\
\hline$\lambda_{\text {ex }}$ for Q / nm & 320 & 320 & 600 \\
Q / \% & $4.94(8) \times 10^{-2}$ & $2.54(3) \times 10^{-2}$ & $9.0(2) \times 10^{-3}$ \\
& & & \\
Lifetime / $\mu \mathrm{s}$ & $4.5(2): 34.1(3) \%$ & $4.8(1): 21.2(5) \%$ \\
& $1.32(3): 65.9(3) \%$ & $0.95(1): 78.8(5) \%$
\end{tabular}

Fig. 3 (a) Corrected and normalized emission spectra of $\mathrm{Yb}^{3+}$-BPDC and LDS $750 @ \mathrm{Yb}^{3+}$-BPDC upon excitation at 320 or $600 \mathrm{~nm}$. Measurements were performed at room temperature on solid samples. (b) Corrected and normalized excitation spectra upon monitoring emission at $980 \mathrm{~nm}$ of $\mathrm{Yb}^{3+}$-BPDC MOFs with and without LDS 750. (c) $\mathrm{Yb}^{3+}$-centred quantum yields and lifetimes (determined upon excitation at $355 \mathrm{~nm}$ and monitoring emission at $980 \mathrm{~nm}$ ) of solid samples of $\mathrm{Yb}^{3+}$-BPDC and LDS 750@ $\mathrm{Yb}^{3+}$-BPDC at room temperature (2 $\sigma$ are given between parentheses). 
of LDS 750@ $\mathbf{Y b}^{3+}$-BPDC is lower by 1.9 times compared to the value recorded for the $\mathbf{Y b}^{3+}$-BPDC. Therefore, the incorporation of LDS 750 inside the pores of $\mathbf{Y b}^{3+}$-BPDC decreases the sensitization efficiency of BPDC towards $\mathrm{Yb}^{3+}$ emission. Quantum yield value of LDS 750@ $\mathbf{Y b}^{3+}$-BPDC recorded upon excitation at $600 \mathrm{~nm}$ (LDS 750 chromophore) decreases to $9.0(2) \times 10^{-3} \%$, suggesting that the sensitization efficiency of LDS 750 is lower than that of BPDC. Inefficient energy transfer from LDS 750 to $\mathrm{Yb}^{3+}$ is further supported by the observation of residual broadband emission along with sharp $\mathrm{Yb}^{3+2} \mathrm{~F}_{5 / 2} \rightarrow{ }^{2} \mathrm{~F}_{7 / 2}$ transition centred at $980 \mathrm{~nm}$ in the emission spectrum of the LDS 750@ $\mathbf{Y b}^{3+}$-BPDC upon $600 \mathrm{~nm}$ excitation (Fig. 3a, red curve). Nevertheless, we demonstrated that by using our approach, the excitation wavelength of $\mathbf{Y b}^{3+}$-BPDC MOF can be tuned by straightforward incorporation of an appropriate chromophore through non-covalent interactions.

\section{Conclusions}

This work constitutes a major achievement towards obtaining $\mathrm{RE}^{3+}$-based NIR-emitting MOFs with controllable luminescent properties. The originality of this approach lies in the noncovalent encapsulation of chromophores in the pores for the sensitization of $\mathrm{RE}^{3+}$ that are part of the MOF scaffold, which broadens the range of chromophore selection for $\mathrm{RE}^{3+}$ sensitization in systems where covalent or dative attachment is difficult or impossible. We have shown that such sensitization was sufficiently efficient with even a small amount of sensitizer to generate a NIR signal from $\mathrm{Yb}^{3+}$ that can be both monitored and quantified. Using this strategy, we have been able to create a MOF that not only emits, but also absorbs in the biological window, which represents a significant breakthrough in the realm of biological imaging with MOFs: thus far, all MOFs, including nanoMOFs that we have tested, absorb in the UV or high energy visible range. Excitation light with such low energy is favourable to biological systems as it minimizes interaction with sensitive samples.

\section{Conflicts of interest}

There are no conflicts to declare.

\section{Acknowledgements}

The MOF synthesis and development work in this project received partial support from the Defense Threat Reduction Agency-Joint Science and Technology Office for Chemical and Biological Defense Basic Research (Grant no. HDTRA1-16-10044, NLR). In addition, this work is partially supported by La Ligue Contre le Cancer, La Region Centre, Agence Nationale de la Recherche (NIRA ANR-13-BS08-0011 and LUMZIF ANR-12BS07-0012). S. P. acknowledges support from the Institut National de la Santé et de la Recherche Médicale (INSERM). The authors thank the Petersen Nano Fabrication and Characterization Facility at the University of Pittsburgh for access to
PXRD instrumentation. The authors thank Dr Kristy Gogick for helpful discussion and Ms. Disi Wang for figure preparation.

\section{References}

1 R. Weissleder, Nat. Biotechnol., 2001, 19, 316.

2 V. J. Pansare, S. Hejazi, W. J. Faenza and R. K. Prud'homme, Chem. Mater., 2012, 24, 812-827.

3 I. Martinić, S. V. Eliseeva and S. Petoud, J. Lumin., 2017, 189, 19-43.

4 S. V. Eliseeva and J.-C. G. Bünzli, Chem. Soc. Rev., 2010, 39, 189-227.

5 S. I. Weissman, J. Chem. Phys., 1942, 10, 214-217.

6 H. Uh and S. Petoud, C. R. Chim., 2010, 13, 668-680.

7 K. A. White, D. A. Chengelis, M. Zeller, S. J. Geib, J. Szakos, S. Petoud and N. L. Rosi, Chem. Commun., 2009, 45064508, DOI: 10.1039/b909658b.

8 K. A. White, D. A. Chengelis, K. A. Gogick, J. Stehman, N. L. Rosi and S. Petoud, J. Am. Chem. Soc., 2009, 131, 18069-18071.

9 J. An, C. M. Shade, D. A. Chengelis-Czegan, S. Petoud and N. L. Rosi, J. Am. Chem. Soc., 2011, 133, 1220-1223.

10 A. Foucault-Collet, K. A. Gogick, K. A. White, S. Villette, A. Pallier, G. Collet, C. Kieda, T. Li, S. J. Geib, N. L. Rosi and S. Petoud, Proc. Natl. Acad. Sci. U. S. A., 2013, 110, 17199-17204.

11 T.-Y. Luo, C. Liu, S. V. Eliseeva, P. F. Muldoon, S. Petoud and N. L. Rosi, J. Am. Chem. Soc., 2017, 139, 9333-9340.

12 J. Della Rocca, D. Liu and W. Lin, Acc. Chem. Res., 2011, 44, 957-968.

13 P. Horcajada, R. Gref, T. Baati, P. K. Allan, G. Maurin, P. Couvreur, G. Férey, R. E. Morris and C. Serre, Chem. Rev., 2012, 112, 1232-1268.

14 Y. Cui, Y. Yue, G. Qian and B. Chen, Chem. Rev., 2012, 112, 1126-1162.

15 D. T. de Lill, A. de Bettencourt-Dias and C. L. Cahill, Inorg. Chem., 2007, 46, 3960-3965.

16 C. Yuanjing, S. Ruijing, Y. Jiancan, L. Min, W. Ziqi, W. Chuande, Y. Yu, W. Zhiyu, C. Banglin and Q. Guodong, Adv. Mater., 2015, 27, 1420-1425.

17 X. Lian and B. Yan, RSC Adv., 2016, 6, 11570-11576.

18 D. X. Xue, A. J. Cairns, Y. Belmabkhout, L. Wojtas, Y. Liu, M. H. Alkordi and M. Eddaoudi, J. Am. Chem. Soc., 2013, 135, 7660-7667.

19 V. Guillerm, L. Weselinski, Y. Belmabkhout, A. J. Cairns, V. D'Elia, L. Wojtas, K. Adil and M. Eddaoudi, Nat. Chem., 2014, 6, 673-680.

20 D. Alezi, A. M. P. Peedikakkal, Ł. J. Weseliński, V. Guillerm, Y. Belmabkhout, A. J. Cairns, Z. Chen, Ł. Wojtas and M. Eddaoudi, J. Am. Chem. Soc., 2015, 137, 5421-5430.

21 Y. J. Li, Y. L. Wang and Q. Y. Liu, Inorg. Chem., 2017, 56, 2159-2164.

22 M. L. Gao, W. J. Wang, L. Liu, Z. B. Han, N. Wei, X. M. Cao and D. Q. Yuan, Inorg. Chem., 2017, 56, 511-517. 